\title{
An application of Bayesian statistics to the extragalactic Cepheid distance scale
}

\author{
Thomas G. Barnes III ${ }^{1}$, Thomas J. Moffett ${ }^{2}$, W.H. Jefferys ${ }^{3}$, \\ Amy D. Forestell ${ }^{3}$ \\ ${ }^{1}$ The University of Texas at Austin, McDonald Observatory, \\ 1 University Station, C1402, Austin, TX 78712-0259, USA \\ ${ }^{2}$ Purdue University, Physics Department, 1396 Physics Bldg., West \\ Lafayette, IN 47907-1396, USA \\ ${ }^{3}$ The University of Texas at Austin, Department of Astronomy, \\ 1 University Station, C1400, Austin, TX 78712-0259, USA
}

\begin{abstract}
We have determined quasi-geometric distances to the Magellanic Clouds, M31 and M33. Our analysis uses a Bayesian statistical method to provide mathematically rigorous and objective solutions for individual Cepheids. We combine the individual distances with a hierarchical Bayesian model to determine the galactic distances. We obtain distance moduli $18.87 \pm 0.07 \mathrm{mag}$ (LMC, 12 stars), $19.14 \pm 0.10$ (SMC, 8 stars), $23.83 \pm 0.35 \mathrm{mag}$ (M33, 1 star) and $25.2 \pm 0.6 \mathrm{mag}$ (M31, 1 star) - all uncorrected for metallicity. The M31 and M33 distances are very preliminary. If the PL relations of the LMC, SMC and Galaxy are identical, our results exclude the metallicity effect in the $V,(V-R)$ surface brightness method predicted by Hindsley \& Bell (1989) at the $5 \sigma$ level. Alternately, if Hindsley \& Bell's prediction is adopted as true, we find a metallicity effect intrinsic to the Cepheid PL relation requiring a correction $\Delta\left(V-M_{V}\right)=(0.36 \pm 0.07) \Delta[A / H]$ mag. The latter has the opposite sign to other observational estimates of the Cepheid metallicity effect.
\end{abstract}

\section{Introduction}

The extragalactic distance scale is founded on the Cepheid PL relation. That relation is built from LMC and Galactic Cepheids using distances largely tied to main-sequence fitting of open clusters. This distance scale is not geometric and may contain a metallicity effect. Our long-term goals are to establish quasigeometric distances to nearby galaxies and to put boundaries on the effect of metallicity on the Cepheid PL relation.

The surface brightness method has recently been calibrated geometrically through interferometric angular diameters of Cepheids (Nordgren et al. 2002). That calibration yields the same parallax for $\delta$ Cep, to $1 \pm 4 \%$, as obtained from a Hubble Space Telescope parallax (Benedict et al. 2002). The new calibration 
thus permits Cepheid distances to be determined that are very close to geometric ('quasi-geometric').

\section{Observations}

Photometry and radial velocities were taken from the literature for the LMC and SMC Cepheids. Photometry acquired in the Cousins system was transformed to the Johnson system. Typically there were about 50 photometric and 40 radial velocity values. We adopted individual reddenings.

Photometry for the M31 and M33 Cepheids was taken from the DIRECT project website (http://cfa-www.harvard.edu/ kstanek/DIRECT/) and transformed to the Johnson system. Radial velocities of Cepheids in M31 and M33 were obtained with the Hobby-Eberly Telescope at McDonald Observatory as described by Forestell et al. (these proceedings). Typically there were about 20 photometric and 10 radial velocity values. We adopted galactic mean reddenings.

\section{Analyses}

\subsection{Bayesian, Markov Chain Monte Carlo analysis}

We used the Bayesian, Markov Chain Monte Carlo statistical method of Barnes et al. (2003) with the surface brightness calibration of Nordgren et al. (2002). Our approach creates the likelihood function, fits Fourier series models to the photometry and radial velocities, adopts appropriate priors, samples the posterior probability distributions of the stellar properties using MCMC (10000 samples), and averages over the various models (such as the orders of the Fourier series) to estimate the quantities of interest (distance, mean absolute magnitude, stellar radius, etc.) and their uncertainties. Because we will be using a hierarchical Bayesian method to combine these distances (in section 3.3), we adopted a flat prior for individual distances in the LMC and SMC. For M31 and M33, with only one Cepheid per galaxy, we adopted an $r^{2} d r$ prior to avoid Lutz-Kelker bias.

\subsection{Corrections for tilt}

To determine the distance to the center of each galaxy, we applied corrections to the individual Cepheid distances that arise from extension of the galaxy along the line of sight. We used the formalism of van der Marel \& Cioni (2001), which requires the host galaxy's inclination $(i)$ to the plane of the sky and the position angle $(p a)$ of its line of nodes. For the LMC we adopted $i=34^{\circ} .7, p a=122.5$; for the SMC, $i=68^{\circ}, p a=148^{\circ}$. Distance corrections fell in the range $-2 \%$ to $+4 \%(\mathrm{LMC}),-5 \%$ to $+3 \%(\mathrm{SMC})$, and were negligible for M31 and M33.

\subsection{Combining the individual distance estimates}

We combined the distance results for the individual Cepheids in the LMC and SMC using a hierarchical Bayes approach (Zaslavsky 2002). This has the advantage of taking into account the distribution of the posterior probabilities for 
the distance to each star as well as their degree of agreement (or disagreement). We regard the 10000 distances $r_{i}$ of each Cepheid, corrected for the extension of the galaxy, as measurements of a quantity which itself has a normal distribution with mean $r$ and standard deviation $\sigma$, to be determined. A prior is placed on $r$, which we take to be proportional to $r^{2}$ in accordance with our prior notion that the number of stars in a shell of thickness $d r$ is proportional to $r^{2} d r$. This avoids Lutz-Kelker bias in our combined distance. The hierarchical Bayesian code was run for 100000 samples to generate the posterior probability distribution for the distance to the center of each galaxy.

\section{Results}

From 12 Cepheids, the distance to the LMC is found to be $59400 \pm 1900 \mathrm{pc}$. This corresponds to a distance modulus $18.87 \pm 0.07 \mathrm{mag}$. From 8 Cepheids in the $\mathrm{SMC}$ we find a distance $67300 \pm 3100 \mathrm{pc}$, i.e., $19.14 \pm 0.10 \mathrm{mag}$. In the cases of M31 and M33, we have only one Cepheid each and their distances do not yet use all the data at hand (see Forestell et al., these proceedings). These distances are quite preliminary: M33: $23.83 \pm 0.35 \mathrm{mag}$; M31: $25.2 \pm 0.6 \mathrm{mag}$.

Using the individual absolute magnitudes, we have formed a Cepheid periodluminosity relation for all four galaxies and compare it to our Galaxy (Fig. 1). It is clear in the PL relation that LMC and SMC Cepheids are fully intermingled, and agree with the PL relation for Galactic Cepheids. The mean displacement in $M_{V}$ of our LMC sample from the Galactic relation is $+0.00 \pm 0.09 \mathrm{mag}$; for the SMC, $-0.12 \pm 0.09$ mag.

Based on model atmospheres, Hindsley \& Bell (1989) predicted that distance moduli obtained by the visual surface brightness relation would require a correction due to metallicity $\Delta\left(V-M_{V}\right)=0.5 \Delta[A / H]$. In the PL relation this implies a correction $\Delta M_{V}=-0.5 \Delta[A / H]$. This prediction has never been verified due to the very small metallicity range of Galactic Cepheids with sufficient data. Adopting Magellanic Cloud metallicities from Luck et al. (1998), $-0.34 \mathrm{dex}$ (LMC) and $-0.68 \mathrm{dex}$ (SMC), Hindsley \& Bell would predict displacements of $\Delta M_{V}=+0.17 \mathrm{mag}$ and $+0.34 \mathrm{mag}$, respectively, relative to the Galaxy. This is not observed in Fig. 1 at the $5 \sigma$ level.

Alternately, if we assume that Hindsley \& Bell's prediction is true, and correct our $M_{V}$ values accordingly, the intrinsic PL relations of the three galaxies would differ. Interpreted as a metallicity effect in the Cepheid PL relation, this would require a metallicity correction to the distance modulus $\Delta\left(V-M_{V}\right)=$ $(0.36 \pm 0.07) \Delta[A / H]$ mag. Such a correction is opposite in sign to that suggested by other observational tests (viz. Freedman et al. 2001).

Acknowledgments. We thank Peter Mueller of M.D. Anderson Medical Center (Texas) for suggesting the hierarchical Bayes approach. This research was supported in part by NSF grants AST-9986817 (TGB) and AST-9987347 (TJM). 


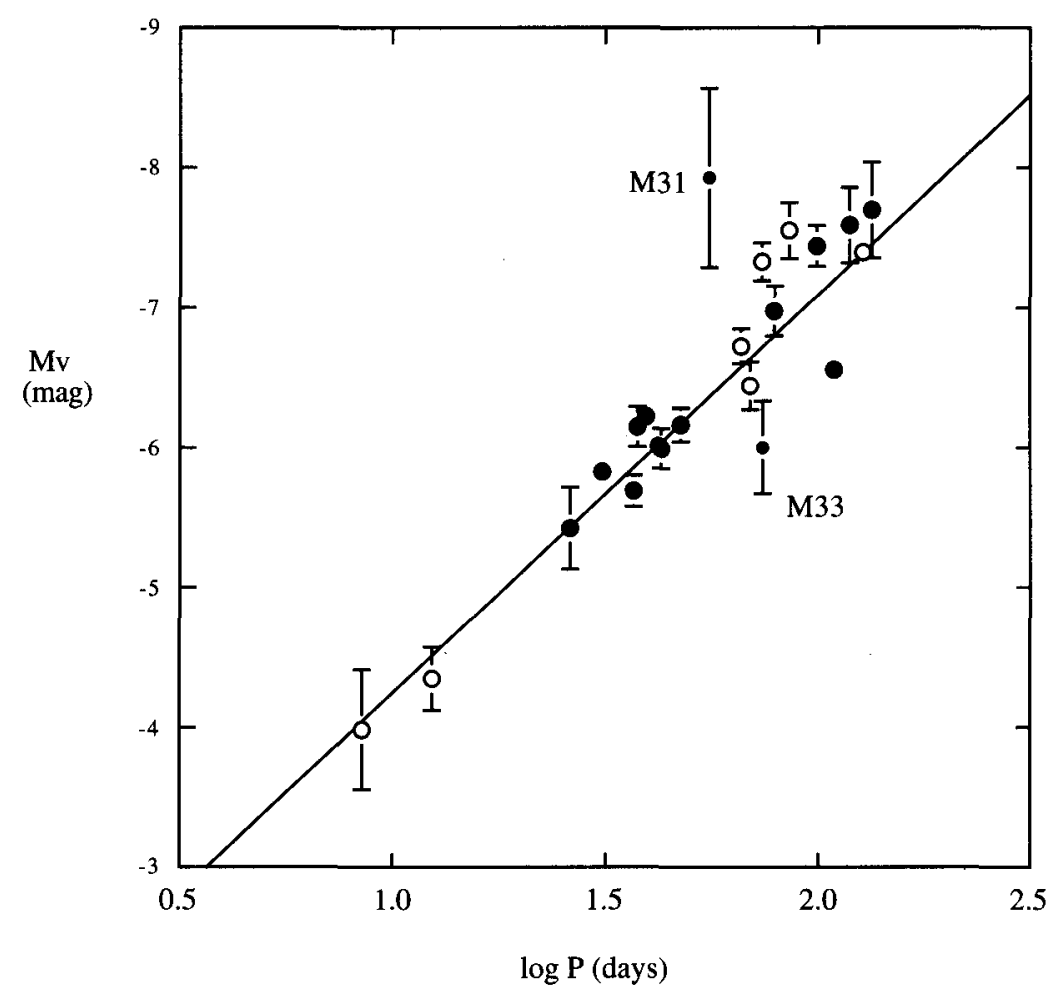

Figure 1. The combined Cepheid PL relation for the LMC, SMC, M31, and M33. The Galactic PL relation is the consensus relation from Barnes et al. (2003). LMC Cepheids are shown by solid symbols; SMC Cepheids by open symbols; M31 and M33 Cepheids are labeled.

\section{References}

Barnes, T.G., Jefferys, W.H., Berger, J.O., Mueller, P.J., Orr, K., Rodriguez, R. 2003, ApJ, 592, 539

Benedict, G.F., et al. 2002, AJ, 124, 1695

Freedman, W.L., et al. 2001, ApJ, 553, 47

Hindsley, R., Bell, R. 1989, ApJ, 341, 1004

Luck, R.E., Moffett, T.J., Barnes, T.G., Gieren, W.P. 1998, AJ, 115, 605

Nordgren, T.E., Lane, B.F., Hindsley, R.B., Kervella, P. 2002, AJ, 123, 3380

van Der Marel, R.P., Cioni, M.-R. 2001, AJ, 122, 1807

Zaslavsky, A.M. 2002 in Chap 14 in "Subjective and Objective Bayesian Statistics", by S.J. Press (Wiley Interscience)) 